\title{
Data Tables for Archaeoastronomy on Inca Sites in the Argentine Northwest
}

\author{
Ivan Leibowicz \\ $\mathrm{MH} I \mathrm{CH} U \mathrm{U}-\mathrm{CONICET}$, Argentina \\ pinocarriaga@hotmail.com
}

Ricardo Moyano

INCIHUSA-CONICET, Argentina mundosubterraneo@yahoo.es

Alejandro Ferrari

CONICET, Argentina

alejandroferra@gmail.com

Félix Acuto

IMHICHU-CONICET, Argentina

facuto@gmail.com

Cristian Jacob

Faculty of Philosophy and Letters, University of Buenos Aires

zamapurro@yahoo.com 
The values of azimuth and height were determined by three observations of the Sun, using a theodolite and GPS, including values of the geographical position and local time.

Parallax calculation: $\mathrm{h}=(\mathrm{hteo}-\mathrm{r})+\mathrm{p}$

$\mathrm{h}=$ corrected height horizon; $\mathrm{hteo}=$ height measured with theodolite; $r=$ atmospheric refraction; $\mathrm{p}=$ parallax $\left(57^{\prime}\right)$

\section{Legend}

$\lambda$

$\varphi$

TU

TT

ET

$\delta$

HA

LHA

masl

$h=$

A

Aube

$r$

obs.

calc.

V

h geographic longitude

geographic latitude

universal time (GPS)

terrestrial dynamical time

equation of time*

declination

hour angle

local hour angle

meters above sea level

Sun height above the horizon

Sun azimuth

object azimuth

atmospheric refraction correction

obs: observed

calc: calculated

theodolite measurement on the

vertical plane

theodolite measurement on the horizontal plane

\section{Acronyms}

P-DSSR pre-December solstices sunrise

DSSR December solstice sunrise

P-DSSS pre-December solstice sunset

DSSS December solstice sunset

P-JSSR pre-June solstice sunrise

JSSR June solstice sunrise

P-JSSS pre-June solstice sunset

JSSS June solstice sunset

$\mathrm{P}$-EQSR pre-equinox sunrise

EQSR equinox sunrise

P-EQSS pre-equinox sunset

EQSS equinox sunset

NMMR northern major standstill moonrise

NMMS northern major standstill moonset

SMMR southern major standstill moonrise

SMMS southern major standstill moonset

NmMR northern minor standstill moonrise

NmMS northern minor standstill moonset

* This is the difference between apparent solar time and mean solar time. In the Almanac it is the reference point for when the sun passes through the $90^{\circ} \mathrm{W}$ meridian. 


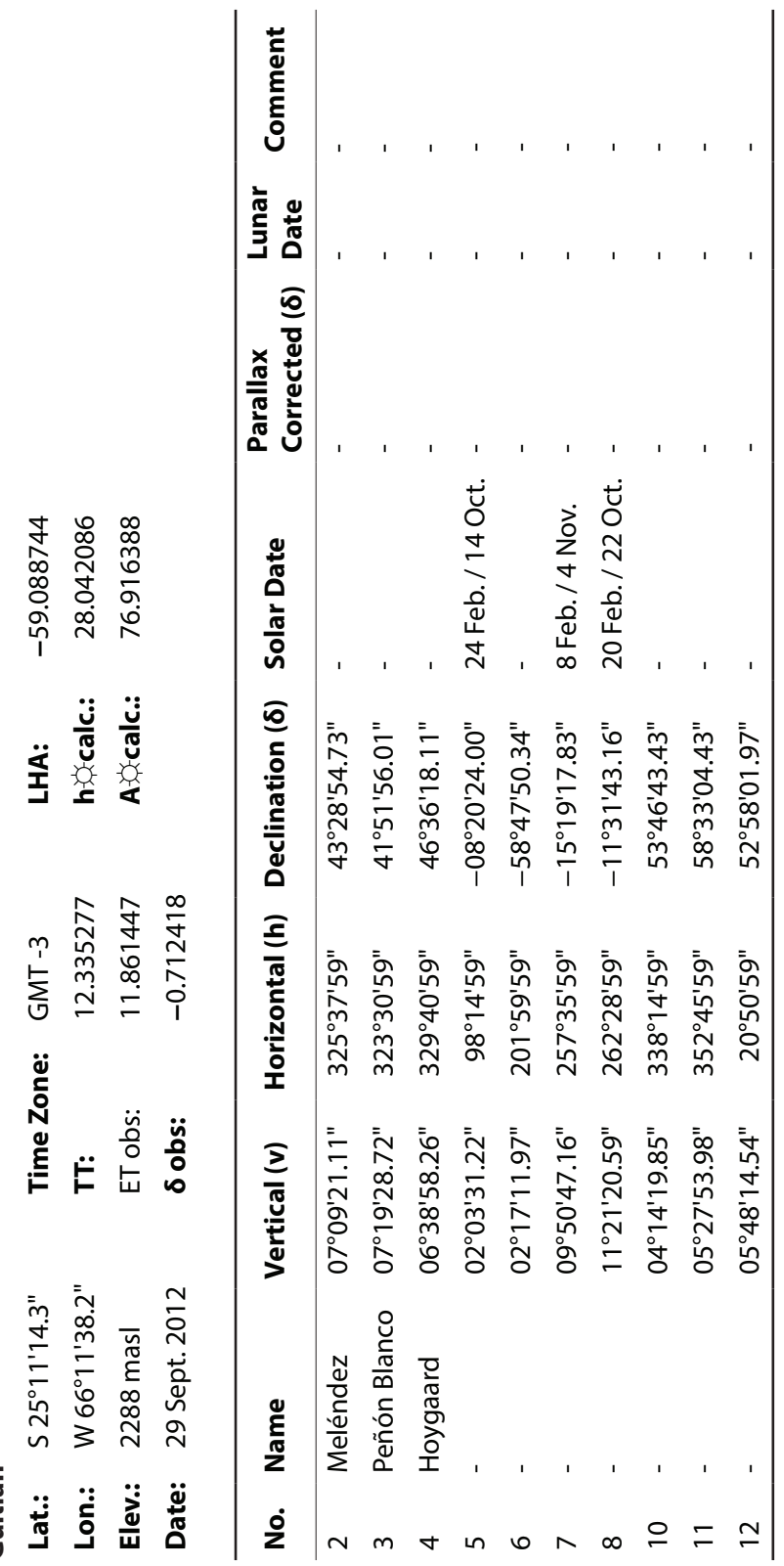

๑) 2017 EQUINOX PUBLISHING LTD 


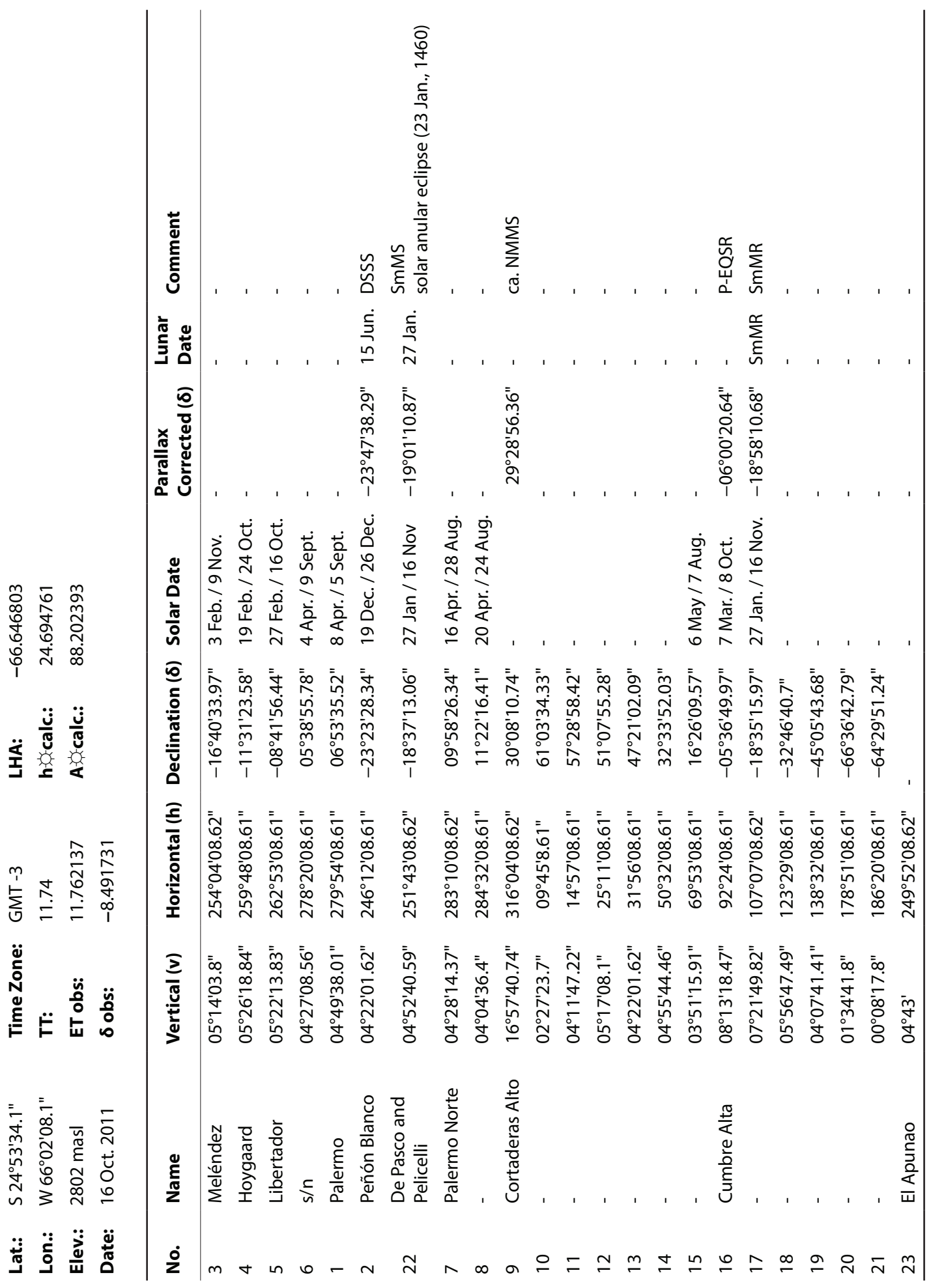




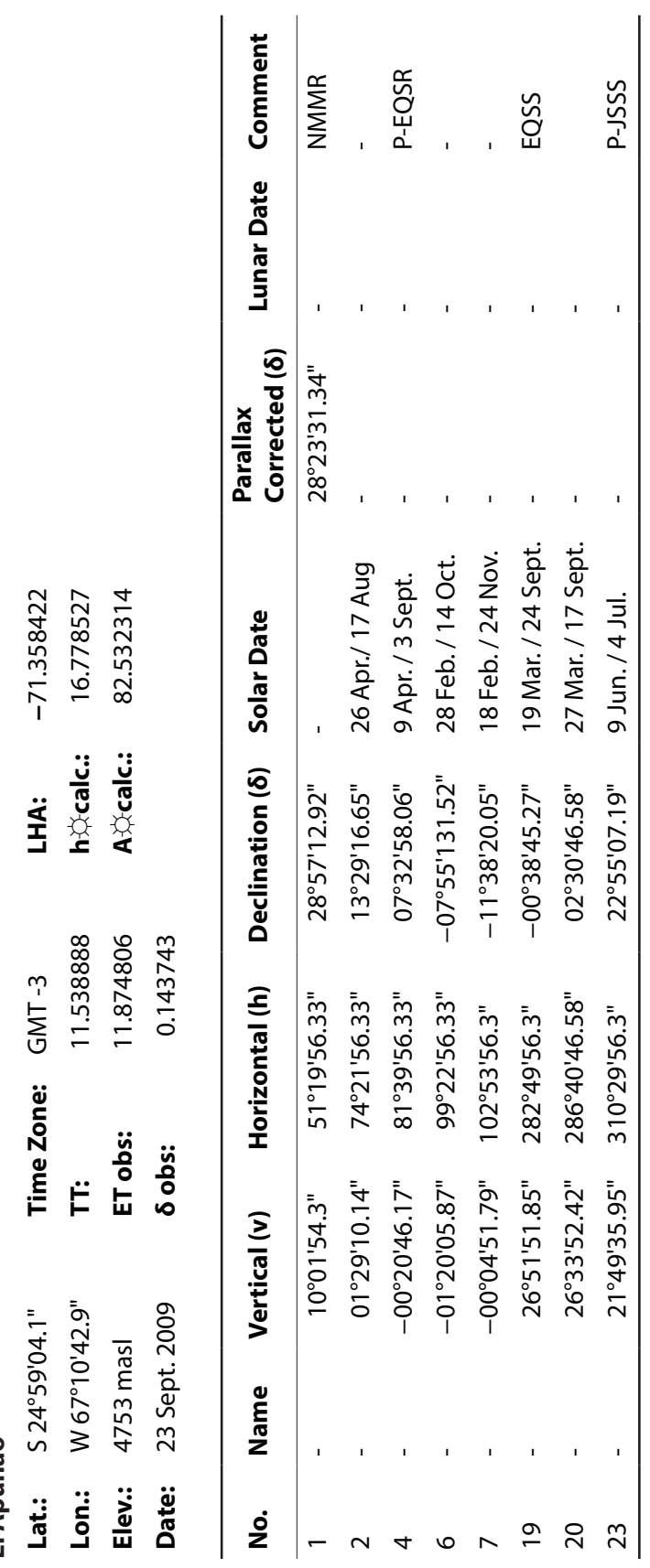

๑) 2017 EQUINOX PUBLISHING LTD 


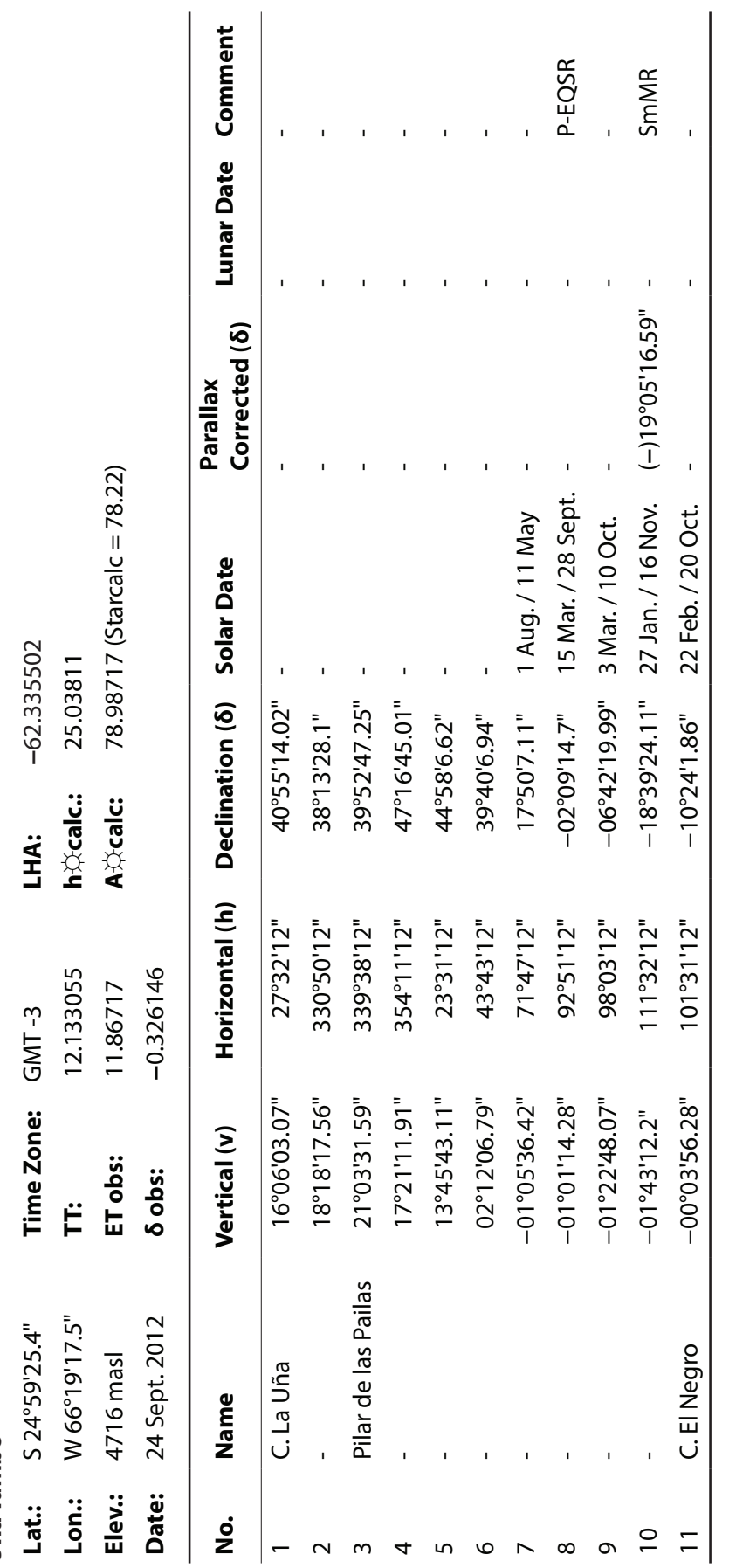

C 2017 EQUINOX PUBLISHING LTD 